\title{
OPTICALLY CONTROLLED MIXING IN MICRODROPLETS
}

\author{
Roman O. Grigoriev* and Michael F. Schatz ${ }^{\dagger}$ \\ Center for Nonlinear Sciences and School of Physics \\ Georgia Institute of Technology, Atlanta, GA 30332-0430
}

\begin{abstract}
$\underline{\text { ABSTRACT }}$
Liquid microdroplets represent a convenient system for studies of mixing by chaotic advection in discrete microscopic volumes. The mixing properties of the flows in microdroplets are governed by their symmetries, which give rise to invariant surfaces serving as barriers to transport. Complete three-dimensional mixing by chaotic advection requires destruction of all flow invariants. To illustrate this idea, we demonstrate that complete mixing can be obtained in a time-dependent flow produced by motion of a microdroplet along a two-dimensional path. The theoretical predictions are confirmed by experiments that optically manipulate and mix microdroplets.
\end{abstract}

\section{INTRODUCTION}

Most microfluidic systems, which are being developed into "labs-on-a-chip" that promise revolutionary applications in biotechnology, chemistry and medicine,${ }^{1-4}$ require efficient mixing of initially distinct fluid volumes. Liquids, however, do not mix easily at the scale of typical microfluidic devices. Physically, microscale flows are characterized by a low Reynolds number $R e \equiv V a / \nu<1$, where $V$ and $a$ are, respectively, a characteristic flow speed and length, and $\nu$ is the liquids' kinematic viscosity. In this regime, flows are laminar, and turbulence, which governs mixing rates in macroscopic systems, cannot arise. Yet the size of typical microfluidic devices is too large for molecular diffusion, which usually governs mixing at smaller scales, to become effective. Thus, efficient mixing of liquids at the microscale requires a stirring mechanism, such as chaotic advection, ${ }^{5,6}$ that stretches and folds fluid elements throughout the entire volume of the flow. The folding leads to a decrease in the average distance between unmixed volumes of liquid with different composition, while stretching sharpens the concentration gradients enhancing diffusion, which acts

\footnotetext{
*Assistant Professor

${ }^{\dagger}$ Associate Professor
}

more rapidly to smooth out remaining nonuniformities. For devices based on continuous flow through microchannels, strategies for inducing chaotic mixing by altering device geometries have been proposed and verified experimentally. ${ }^{7,8}$

Our focus here will be on discrete volume systems ${ }^{4,9-11}$ which allow miniaturization of many standard laboratory protocols that are difficult to realize with continuous flow. We will concentrate primarily on spherical volumes, such as microdroplets of one liquid suspended in another liquid, both because such configurations are easy to implement and study experimentally and because the flows inside spherical volumes can be computed analytically due to the high symmetry of the problem. This high symmetry, coupled with the time-reversal invariance of the Stokes (low-Re) flow, proves to be a mixed blessing as it also makes designing a chaotic microflow with good mixing properties a lot more challenging than in the case of unbounded geometries. Below we discuss the existing theoretical and experimental results on mixing in microdroplets prior to describing our own research.

\section{Theoretical Results}

The three most common flows that arise in spherical microdroplets are the Hill's spherical vortex (or "dipole") flow, the axisymmetric extensional (or "quadrupole") flow, and the Taylor (or "rolling") flow. The dipole flow was originally computed by Hadamard and describes the flow inside a viscous droplet undergoing translational motion due to buoyancy, ${ }^{12}$ thermocapillary effect ${ }^{13}$ or nonuniform electric field. ${ }^{14}$ The corresponding velocity field is given (in the frame of the drop) by

$$
\mathbf{v}_{d}=V_{d} a^{-2}\left[(\mathbf{e} \cdot \mathbf{r}) \mathbf{r}-\left(2 r^{2}-a^{2}\right) \mathbf{e}\right]
$$

where $a$ is the droplet diameter, e is a unit vector in the direction of motion and $V_{d}$ is the characteristic velocity (Fig. 1). The quadrupole flow (Fig. 2)

$$
\begin{aligned}
\mathbf{v}_{q}=V_{q} a^{-3} & {\left[\left(r^{2}+2(\mathbf{e} \cdot \mathbf{r})^{2}-a^{2}\right) \mathbf{r}\right.} \\
& \left.-\left(5 r^{2}-3 a^{2}\right)(\mathbf{e} \cdot \mathbf{r}) \mathbf{e}\right],
\end{aligned}
$$




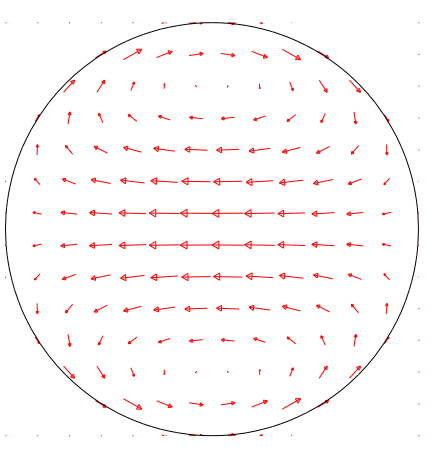

Figure 1: Dipole flow at the mid-plane of the droplet. Vector $\mathbf{e}$ is in the horizontal direction.

with characteristic velocity $V_{q}$, can be induced by placing the droplet in an axisymmetric extensional flow $^{15} \hat{\mathbf{v}}^{\infty} \propto \mathbf{r}-3(\mathbf{e} \cdot \mathbf{r}) \mathbf{e}$, or subjecting it to a uniform electric field. ${ }^{16}\left(\hat{\mathbf{v}}^{\infty}\right.$ denotes the flow far from the droplet.) Finally, the velocity field obtained by placing the droplet in a shear flow $\hat{\mathbf{v}}^{\infty} \propto\left(\mathbf{e}_{1} \cdot \mathbf{r}\right) \mathbf{e}_{2}$, with $\mathbf{e}_{1}$ and $\mathbf{e}_{2}$ two orthogonal unit vectors, has been computed by Taylor ${ }^{15}$ and takes the form

$$
\begin{array}{r}
\mathbf{v}_{t}=V_{t} a^{-3}\left[\left(5 r^{2}-3 a^{2}\right)\left\{\left(\mathbf{e}_{1} \cdot \mathbf{r}\right) \mathbf{e}_{2}+\left(\mathbf{e}_{2} \cdot \mathbf{r}\right) \mathbf{e}_{1}\right\}\right. \\
\left.-4\left(\mathbf{e}_{1} \cdot \mathbf{r}\right)\left(\mathbf{e}_{2} \cdot \mathbf{r}\right) \mathbf{r}+2 a^{2}(1+\lambda)\left(\mathbf{e}_{1} \times \mathbf{e}_{2}\right) \times \mathbf{r}\right],
\end{array}
$$

where $\lambda=\mu / \hat{\mu}$ is the ratio of inside to outside fluid dynamic viscosities and $V_{t}$ is again the characteristic velocity of the flow (Fig. 3). In the limit $\lambda \rightarrow \infty$ this flow reduces to a rigid body rotation of the droplet around the axis $\mathbf{e}_{1} \times \mathbf{e}_{2}$.

If one neglects diffusion, the advection becomes the only transport mechanism, so the trajectories of infinitesimal fluid volumes with different composition are described by the flow

$$
\dot{\mathbf{r}}=\mathbf{v}(\mathbf{r}) \text {. }
$$

The mixing properties of the flow can therefore be defined by the geometrical properties of the invariant sets of the dynamical system (4). The high degree of symmetry of many typical flows leads to the existence of invariants, ${ }^{17}$ which are functions of coordinates that are constant along streamlines of the flow. Each invariant defines, inside the volume of droplet, surfaces on which the flow is effectively two-dimensional. Additional invariants further reduce the flow dimensionality; e.g., flow with two invariants is effectively one-dimensional. For instance, the symmetry of the flows (1)-(3) is so high that each possesses two invariants. As a result all trajectories are periodic and the flows have very poor mixing properties. According to a well known result of dynamical systems theory, chaotic trajectories required for thorough mixing can only be obtained if

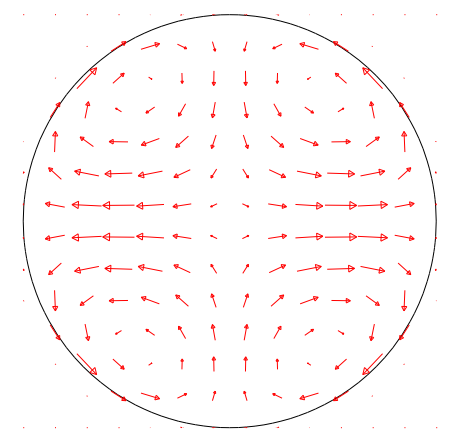

Figure 2: Quadrupole flow at the mid-plane of the droplet. Vector $\mathbf{e}$ is in the horizontal direction.

the effective dimensionality of the flow is at least three.

In recognition of this fact, most theoretical investigations have concentrated on either analytical ${ }^{18-21}$ or numerical ${ }^{22-25}$ studies of combinations of the above flows and introduction of time-dependence, with both approaches targeted at increasing the effective dimensionality of the flow. Most of the theoretical progress in understanding chaotic mixing in bounded flows has been achieved via perturbative studies of steady axisymmetric flows (1) and (2), which can be easily written in Hamiltonian form by using their invariants. Both flows are structurally unstable: arbitrarily small perturbations can lead to chaos. The effect of small perturbations can be included by using a standard perturbation analysis based on averaging their contribution over one period of the unperturbed trajectories. ${ }^{17,20,21}$

However, small perturbations typically only lead to weak nonintegrability, i.e., at onset the mixing occurs inside very thin shells surrounding the unperturbed trajectories and as such is quite ineffective. $^{20}$ Even in the exceptional cases (such as the quadrupole flow combined with slow rotation) when the integrability is destroyed completely, it takes an extremely long time for the chaotic streamline to "diffuse" throughout the droplet. ${ }^{19}$ Quick and thorough mixing inside the droplet is expected to require nonperturbative corrections (i.e., superposition of two or more flows with different symmetries and similar strength). To date, theoretical studies of nonperturbative effects have been limited only to numerical simulations.

\section{Experimental Demonstrations}

With a single exception provided by the recent experimental investigation by Ward and Homsy, ${ }^{26}$ theoretical and experimental research in this area have by and large been completely disconnected. Few of 


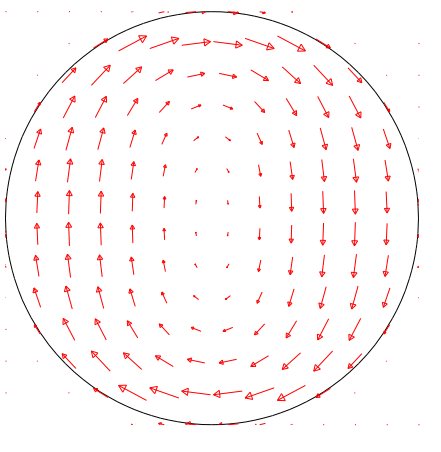

Figure 3: Taylor flow at the mid-plane of the droplet. Vectors $\mathbf{e}_{1}$ and $\mathbf{e}_{2}$ are in the horizontal and vertical direction, respectively. In this example $\lambda=1$.

the theoretical investigations mentioned above are of practical significance, as most of the studied combinations of flows are nearly impossible to realize experimentally. Respectively, the experimental studies of mixing in microdroplets ${ }^{27-32}$ have in no way relied on the existing theoretical results.

Although chaos can, in principle, arise in steady three-dimensional flows, ${ }^{19,20,24}$ all experiments, without exception, relied on time-dependent flows to generate chaotic advection. In one group of experiments ${ }^{28-31}$ deformed microdroplets confined between two flat surfaces were used. Two droplets (one dyed and one un-dyed) were merged and moved by using electrowetting. Regular colored ${ }^{30,31}$ and fluorescent $^{28,29}$ dyes were used to visualize the mixing process. Although detailed studies of the distribution of mixed volumes inside the droplet have not been conducted (the depth-averaged signal has been recorded) it was determined that one-dimensional "shaking" of the droplet does not lead to mixing due to the time-reversibility of the Stokes flow - moving the droplet to the original position restores the initial (unmixed) state. ${ }^{28}$ However, moving the droplet in two dimensions, e.g., around the perimeter of a rectangle, ${ }^{29,30}$ appears to mix the dye better. Similarly, the studies of liquid droplets (or "plugs") confined by microchannels also found that bending the microchannels in two dimensions improves mixing. ${ }^{32}$

A crude two-dimensional model was proposed by Fowler et al. ${ }^{30}$ to explain mixing in droplets moved along square paths (the corresponding experiments were performed with droplets distorted so strongly, they were effectively two-dimensional). Without explicitly referencing the chaotic advection mechanism, the authors suggested that motion in a straight line leads to stretching of the fluid volumes, while switching the direction of motion leads to folding. This stretching and folding repeated multiple times describes a classical chaotic mixing mechanism leading to fractal structure of chaotic invariant sets.

Stretching and folding of fluid elements in spherical droplets has also been demonstrated experimentally ${ }^{26}$ supporting the results of the theoretical analysis ${ }^{25}$ conducted earlier. The primary (dipole) flow in the droplet was generated due to its motion as the droplet sank to the bottom of the container. An additional time-dependent quadrupole flow with the same axis was superimposed by applying alternating vertical electric field. However, the limitations of the experimental setup prevented the thorough mixing of the injected fluorescent dye inside the droplet. As we will show below, this is due to the remaining axial symmetry of the flow which only possesses twodimensional chaotic invariant sets.

\section{FLOW MANIPULATION}

As we have seen so far, chaotic advection requires a flow with rather special properties to be induced inside the microdroplet. The list of "building blocks" for such a flow, however, is not large, regardless of what mechanism is actually used to drive the flow. Detailed experimental studies of different combinations of basic flows, therefore, require considerable flexibility in the experimental setup. Electrical or thermal fields provide arguably the easiest and most flexible way for controlling the flow. Several approaches based on applying the electric field were discussed in the previous section.

\section{$\underline{\text { Experimental Setup }}$}

Here we describe an alternative approach to driving microflows based on the temperature dependence of surface tension (the Marangoni or thermocapillary effect). Manipulating surface tension provides a natural approach to regulating flows at small scales because surface forces, like surface tension, dominate when the surface-to-volume ratio is large. The surface tension at the interface between immiscible fluids can be conveniently altered by changing the temperature; for pure fluids, the surface tension decreases as the temperature increases. The fluids move when gradients in temperature induce surface tension differences. At small scale, even small thermal gradients can cause substantial fluid movement. As a consequence, the thermocapillary effect has been utilized successfully in prototype devices for manipulating tiny quantities of fluid. ${ }^{3,4,33}$ In these devices, temperature variations have been generated with heating/cooling devices (e.g., resistive heaters) placed in physical contact with the fluids. We have recently demonstrated that surface-tension 


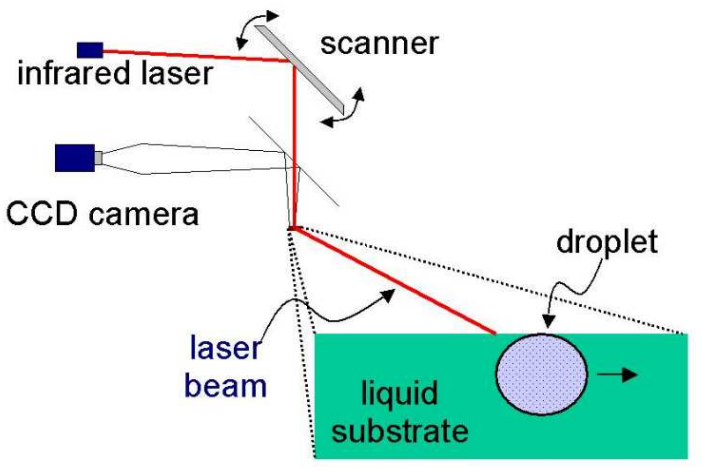

Figure 4: Experimental setup.

gradients can be controlled optically. ${ }^{34}$ Radiative heating of the interface using an intensity-modulated light beam provides a flexible, rapidly-reconfigurable method of driving microflows on substrates with no moving parts and no need to construct on-chip devices (e.g., microscale pipes, valves, etc.) for fluid handling. In particular, microflows can be driven on smooth, featureless solid or liquid substrates.

We use this thermo-optical approach for manipulating the flow inside and outside of microdroplets suspended on an immiscible liquid substrate. Aqueous droplets $(60 \%$ glycerol - $40 \%$ water with $\nu=0.12$ $\mathrm{cm}^{2} / \mathrm{s}$ and density $\left.\rho=1.1 \mathrm{~g} / \mathrm{cm}^{3}\right)$ of radius $a$ $(30 \mu \mathrm{m}<a<3 \mathrm{~mm})$ are immersed in a perfluorocarbon fluid (Fluorinert FC-70 with $\nu=0.14$ $\mathrm{cm}^{2} / \mathrm{s}$ and $\left.\rho=1.9 \mathrm{~g} / \mathrm{cm}^{3}\right)$ substrate of constant thickness $h=4 \mathrm{~mm}$ (see Fig. 4). Typically, droplets float near the substrate-air interface because the substrate is more dense than pure water. The droplets are driven along the substrate surface by temperature-induced surface tension gradients produced by illuminating the substrate with a beam from a tunable infrared $\mathrm{CO}_{2}$ laser (Synrad Model 48G-2-28W with a wavelength range of $9.2-10.7 \mu \mathrm{m})$. The laser's operating wavelength is selected so that the beam is strongly absorbed by the substrate, thereby impressing temperature variations along the free surface; these temperature fields are measured using a liquid-nitrogen-cooled InSb infrared imager (Amber/Raytheon Model 4128). The temperature differences, which are significant only within the vicinity of the beam, induce surface tension gradients via thermocapillarity. A computer-controlled scanning system (LSDI Turbotrak/Pangolin QM2000 controller with GSILumonics G120D galvo-mirrors) permits repositioning of the laser beam at up to 30,000 independent locations per second (with $3 \mu \mathrm{m}$ resolution). Thus, by rapid scanning of the laser beam, surface-tension

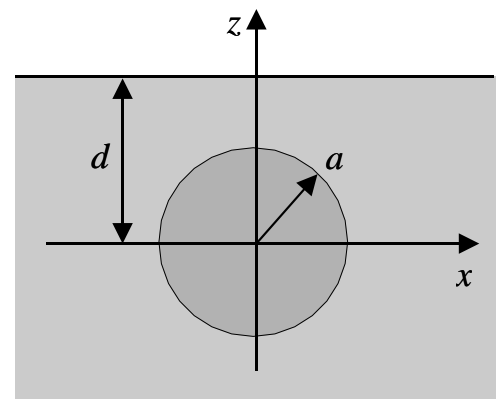

Figure 5: Microdroplet in a liquid substrate. For convenience we will choose the $z$-axis in the vertical direction and place the origin in the center of the droplet.

gradients may be imposed at will at any location on the substrate; these surface forces drive wellcontrolled, localized flows both within and around the droplets. In this way, time-dependent switching of the microdroplet's motion can be implemented. The visualization is performed by illuminating the fluorescent dye inside the droplet with either an extended beam or a thin light sheet from an argon ion laser. A simple illuminator that produces light sheets of $\sim 100 \mu \mathrm{m}$ thickness ${ }^{35}$ has been adapted to a microscope and used in some experiments to determine the uniformity of mixing inside the droplet.

\section{Theoretical Model}

In describing the flow inside the droplet subjected to a nonuniform temperature field we will make a number of simplifying assumptions to obtain a tractable analytical model. First of all, we will assume that the droplet is neutrally buoyant and floats a distance $d>a$ below the substrate/air interface (see Fig. 5) and consider the substrate to be semiinfinite (in other words, $h \gg a$ ). We will assume the droplet to be spherical, which is a good approximation for sufficiently small droplets and the substrate/air interface to be perfectly flat. Next, we will assume that the thermal properties of the liquid inside and outside the droplet are the same (this assumption is non-essential and very easy to lift). We will also assume that the convective heat flux is negligible, so the temperature and velocity fields can be computed independently. Finally, the flow is assumed to be in the Stokes regime.

Neglecting the proximity of the substrate/air interface, the velocity inside and outside the droplet can be found by solving the Stokes equation

$$
\nabla p=\mu \nabla^{2} \mathbf{v}, \quad \nabla \hat{p}=\hat{\mu} \nabla^{2} \hat{\mathbf{v}}
$$


in an infinite domain subject to the incompressibility $\nabla \cdot \mathbf{v}=\nabla \cdot \hat{\mathbf{v}}=0$ and boundary conditions

$$
\begin{gathered}
\mathbf{t} \cdot \mathbf{v}=\mathbf{t} \cdot \hat{\mathbf{v}}, \quad \mathbf{n} \cdot \mathbf{v}=\mathbf{n} \cdot \hat{\mathbf{v}}=0 \\
\mathbf{n} \cdot(\sigma-\hat{\sigma}) \cdot \mathbf{t}=\tau_{s}(\mathbf{t} \cdot \nabla T)
\end{gathered}
$$

on the surface of the droplet $r=a$. In the above expressions $p, \sigma, T$ and $\tau_{s}=\partial \gamma_{s} / \partial T$ denote the pressure, stress tensor, temperature and temperature coefficient of the surface tension, respectively, the hat denotes the quantities pertaining to the outside liquid, the index $s$ denotes the (spherical) surface of the droplet, and $\mathbf{n}$ and $\mathbf{t}$ are unit vectors normal and tangential to the surface. The velocity field can be found by substituting Lamb's general solution ${ }^{36}$ into (6).

As the droplet is constrained to move in the horizontal plane we will only consider the motion arising due to the horizontal component of the temperature gradient produced by the absorption of the laser radiation in the substrate. Assuming the gradient to be uniform in the vicinity of the droplet, $T=\kappa_{0}(\mathbf{e} \cdot \mathbf{r})$, the flow inside the droplet can be easily found and is given ${ }^{13}$ by (1) with $V_{d}=$ $-\kappa_{0} a \tau_{s} /[\mu(2 \lambda+3)]$. The nonuniformity of the temperature gradient will produce a correction to this basic dipole flow. In particular, for a temperature profile quadratic in the distance from the droplet center, $T=\kappa_{1}(\mathbf{e} \cdot \mathbf{r})^{2}$, we find the correction given by a quadrupole flow (2) with $V_{q}=\kappa_{1} a^{2} \tau_{s} /[5 \mu(1+\lambda)]$.

The proximity of the substrate/air interface will produce additional corrections. First of all, due to the thermocapillary effect at that interface induced by the temperature gradient a shear flow will be established in the liquid substrate. The uniform temperature gradient $T=\kappa_{0}(\mathbf{e} \cdot \mathbf{r})$ will induce a uniform (far from the droplet) shear which can be found from the boundary conditions at the substrate/air interface

$$
\mathbf{n} \cdot \hat{\mathbf{v}}=0, \quad \mathbf{n} \cdot \hat{\sigma} \cdot \mathbf{t}=\tau_{p}(\mathbf{t} \cdot \nabla T),
$$

where the index $p$ denotes the (plane) interface. This results in the velocity profile

$$
\hat{\mathbf{v}}^{\infty}=-\frac{\kappa_{0} \tau_{p}}{\hat{\mu}}\left(\mathbf{e}_{z} \cdot \mathbf{r}\right) \mathbf{e}+\hat{\mathbf{v}}_{0} \mathbf{e}
$$

This shear flow leads to a Taylor flow correction (3) with $V_{t}=\kappa_{0} \tau_{p} a /[4 \mu(1+\lambda)]$ and $\mathbf{e}_{1}=\mathbf{e}_{z}, \mathbf{e}_{2}=\mathbf{e}$. A quick comparison of the characteristic velocities $V_{d}$ and $V_{t}$ shows that the shear-induced flow inside the droplet is of roughly the same magnitude as the dipole flow. The superposition of these flows is not chaotic when the vorticity of the shear flow far from the droplet is orthogonal to the axis of the dipole flow, as numerical calculations of Bryden and Brenner ${ }^{24}$ show. This is exactly the case here, as the vorticity of the flow (8) is $\omega=\nabla \times \hat{\mathbf{v}}^{\infty} \propto \mathbf{e}_{z} \times \mathbf{e} \perp \mathbf{e}$.

The last term in (8) describes the mean flow in the substrate. For a liquid substrate of thickness $h$ the mean flow leads to the overall migration of the droplet in the direction away from the hot spot produced by the laser beam with velocity $\hat{\mathbf{v}}_{0}=-\left(\kappa_{0} \tau_{p} / \hat{\mu}\right)(h-d)$, which can be easily found using the no-slip boundary condition at the bottom of the substrate layer. For droplets floating near the top interface, $d \ll h$, this motion is dominant compared with the thermocapillary migration ${ }^{13}$ with speed $(2 / 3) V_{d}$ in the opposite direction. The laser beam will, therefore, repel the droplet.

The proximity of the substrate/air interface will also generate perturbative corrections to the flow inside and outside the droplet. Consider, for instance, the flow outside the droplet due to the applied uniform temperature gradient $T=\kappa_{0}(\mathbf{e} \cdot \mathbf{r})$. The velocity field in the unbounded substrate again follows from the Lamb's general solution. In the stationary reference frame one obtains

$$
\hat{\mathbf{v}}_{d}=-V_{d} \frac{a^{3}}{3 r^{5}}\left[3(\mathbf{e} \cdot \mathbf{r}) \mathbf{r}-r^{2} \mathbf{e}\right] .
$$

The solution satisfying the homogeneous version of the boundary conditions (7) at the substrate/air interface can be found using the method of reflections, which generates a series expansion for the velocity field in powers of $\delta=a / d$ by subsequent reflections of the velocity field $\hat{\mathbf{v}}$ about the plane and spherical interface. ${ }^{37}$ The advantage of this technique compared with, say, solution in bi-spherical coordinates $^{38}$ is that each term in the series preserves the symmetry of the flow. The procedure is straightforward although algebraically intensive, so we will just quote a few of the corrections we have computed.

The leading order correction to the dipole flow (1) corresponds to a rigid translation of the droplet with speed

$$
\mathbf{v}_{d}^{(1)}=\delta^{3} \frac{V_{d}}{24} \mathbf{e} .
$$

The next leading order correction for the flow inside the droplet (in the co-moving reference frame) is

$$
\begin{aligned}
\mathbf{v}_{d}^{(2)} & =\frac{\delta^{4} V_{d}}{32(1+\lambda) a^{3}}\left[-4\left(\mathbf{e}_{z} \cdot \mathbf{r}\right)(\mathbf{e} \cdot \mathbf{r}) \mathbf{r}\right. \\
& \left.+\left(5 r^{2}-3 a^{2}\right)\left(\left(\mathbf{e}_{z} \cdot \mathbf{r}\right) \mathbf{e}+(\mathbf{e} \cdot \mathbf{r}) \mathbf{e}_{z}\right)\right]
\end{aligned}
$$

The corrections to the quadrupole and Taylor flows can be computed in the same manner. For instance, 
we again find the leading order correction to the Taylor flow to be cubic in $\delta$

$$
\mathbf{v}_{t}^{(1)}=\frac{\delta^{3}(2+5 \lambda)}{16(1+\lambda)} \mathbf{v}_{t} .
$$

Higher order corrections are too lengthy to be quoted here. None of the corrections we have computed, however, change the symmetry of the basic flows (1)(3) with respect to the mid-planes of the droplet perpendicular to the directions $\mathbf{e}, \mathbf{e}_{z}$ and $\mathbf{e} \times \mathbf{e}_{z}$.

\section{RESULTS AND DISCUSSION}

Now having understood the effect of optical heating on the motion and flows inside the droplet, we move on to the mixing problem. The mixing properties of the flows in the microdroplet are governed by their symmetries, which give rise to invariant surfaces serving as barriers to transport. Since the flow cannot cross invariant surfaces, the existence of invariants is highly undesirable in the mixing problem as their presence inhibits complete stirring of the full microdroplet volume by chaotic advection. Thus, the key to achieving effective chaotic mixing in a microdroplet (indeed, in any laminar microflow) is to ensure that all flow invariants are destroyed.

\section{Theory}

We will next explore the mixing properties of the flow inside the microdroplet arising due to its motion in the experimental setup described above. To begin with, consider the motion caused by a uniform horizontal temperature gradient (we will take it to be in the $x$-direction). Assuming the substrate to be unbounded, the temperature gradient will induce a dipole flow (1) inside the droplet with the axis $\mathbf{e}=\mathbf{e}_{x}$. The poor mixing properties of this flow can be demonstrated by following the motion of a small dyed fluid element inside the microdroplet (Fig. 6a-c). To determine the evolution of the dyed element, the flow (1) is integrated forward in time starting from an initial condition (Fig. 6a). The dye is quickly stretched along the axis of the flow and along the surface of the droplet within a single characteristic turnover time for the flow (Fig. 6b), but never spreads throughout the droplet, even after repeated stretching (Fig. 6c). Poor mixing can be expected in this case because the steady dipole flow is effectively one-dimensional (and, therefore, cannot be chaotic) because it possesses two invariants ${ }^{17}$

$$
\begin{aligned}
\phi_{d} & =\frac{z}{y}, \\
\psi_{d} & =z^{2}\left(a^{2}-r^{2}\right),
\end{aligned}
$$

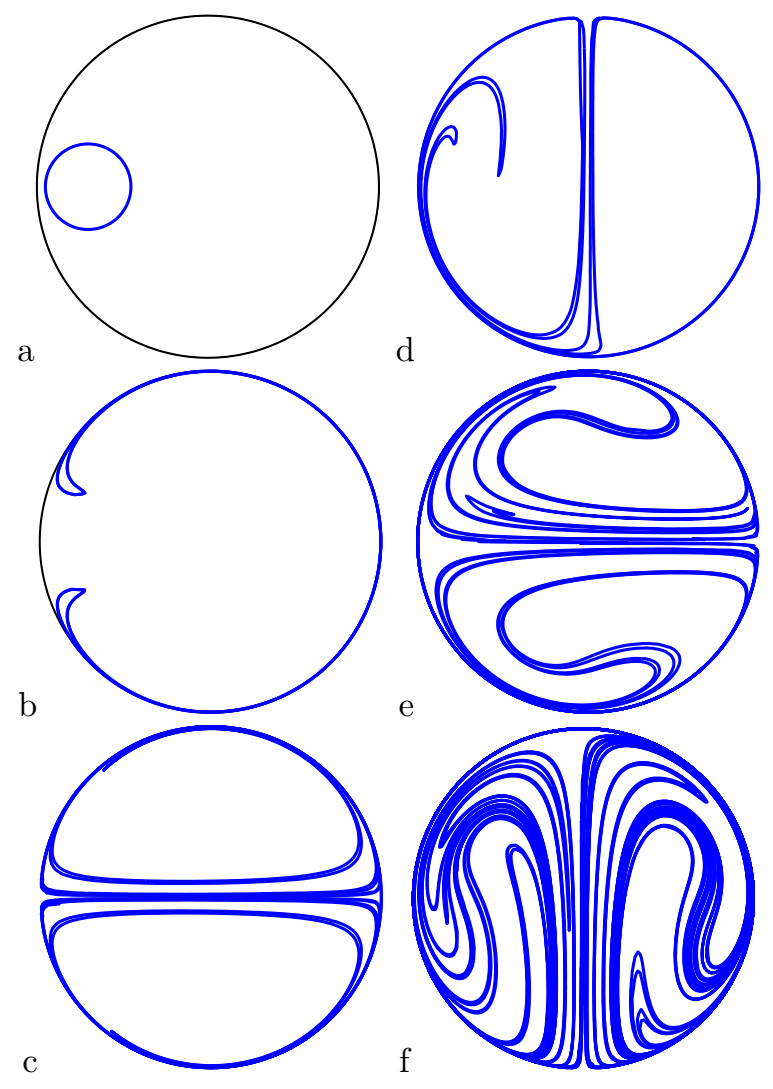

Figure 6: Advection of dye by the dipole flow. The blue line represents the mid-plane cross section $(z=0)$ of the dyed fluid element. The $x$ axis is horizontal and the $y$ axis is vertical. The initial state (a) and stretching in steady dipole flow at $t=6(\mathrm{~b})$ and $t=24$ (c). Stretching and folding in a time-periodic flow obtained by rotating its direction by $90^{\circ}$ in the horizontal plane every 6 time units: the dyed element is shown at $t=12(\mathrm{~d}), t=18$ (e) and $t=24$ (f). Time is measured in units of $a / V$.

related to the orientation of a plane containing the streamline and the stream function of the flow in that plane.

This result can be immediately generalized to droplets confined by straight microchannels. Even though the droplet in this case is not spherical, the flow is topologically identical to the dipole flow shown in Fig. 1. As a result no chaotic advection will result from recirculation of the liquid caused by translation of the droplet, despite claims of apparent good mixing. ${ }^{27}$

It is easy to see that the symmetry of our problem about the $y=0$ mid-plane of the droplet will prevent mixing between the left $(y>0)$ and right $(y<0)$ hemispheres of the droplet, even if the corrections to the dipole flow, such as the quadrupole flow (2) or Taylor flow (3) are included. Indeed, none 
of the streamlines can cross the symmetry plane of the flow, which serves as a barrier to transport in this case. This conclusion clearly also applies to one-dimensional motion of deformed (non-spherical) droplets confined by channels ${ }^{27}$ and flat surfaces. ${ }^{30}$ The apparently uniform mixing observed in those cases is due to depth averaging and hence is misleading. The only way to induce true chaotic mixing in the volume of the droplet is, therefore, by switching the direction of the flow, as was discovered by trial-and-error in earlier electrowetting mixing studies. ${ }^{29,30}$ The pressure-driven flow in liquid droplets moving through bent channels ${ }^{32}$ also undergoes direction switching.

Each flow invariant defines an infinite set of invariant surfaces, much like the $y=0$ symmetry plane. To realize complete mixing, all invariants of the flow must be destroyed. The time-dependence of the flow introduced by switching the direction of the thermal gradient in the horizontal plane plays two different positive roles. First of all, it destroys the invariant $\phi_{d}$ of the dipole flow. Second, it increases the effective dimensionality of the flow by one. The resulting flow, therefore, becomes effectively threedimensional (two space variables plus time) and so chaotic dynamics becomes possible. For instance, repeated stretching and folding of the fluid elements by a time-periodic flow produces efficient stirring in the $z=0$ mid-plane of the droplet, as the results of numerical integration shown in Figs. 6a,d-f demonstrate. This periodic flow can be thought of as a three-dimensional version of the two-dimensional Aref's blinking vortex. ${ }^{5}$

It is important to note that the presence of chaotic advection does not guarantee good mixing. Indeed, the invariant $\psi_{d}$ is not destroyed by direction switching, so that chaotic flow is confined to twodimensional surfaces of revolution defined by

$$
x^{2}+y^{2}=a^{2}-z^{2}-\frac{\psi_{d}}{z^{2}},
$$

as the Poincaré section of the flow (Fig. 7a) indicates. Under the action of chaotic advection the dyed fluid element will only spread over thin shells defined by equation (14), where $\psi_{d}$ varies over the values corresponding to the initially dyed region of the fluid. Clearly, the same conclusions apply if the axis of the flow is rotated in a plane by arbitrary angles at arbitrary times (or if the axis oscillates periodically as proposed by Angilella and Brancher ${ }^{21}$ ). This result indicates that the symmetry of the pure dipole flow is so high that mixing will be incomplete even with the addition of time-dependence.

Next we need to incorporate the effects of the

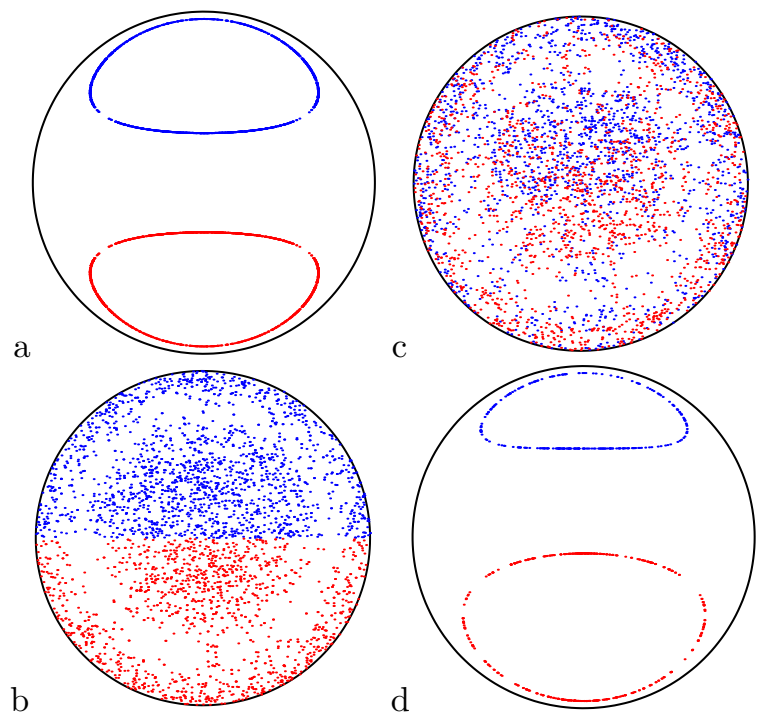

Figure 7: Poincaré sections of dye particles crossing the $x=0$ mid-plane of a droplet. The $z$ axis is vertical. Dye particles that are initially in the top hemisphere $(z>0)$ are shown in blue while dye particles that are initially in the bottom hemisphere $(z<0)$ are shown in red. The flow is made time dependent by switching its direction by $54^{\circ}$ every 6 time units. (a) Dipole flow. (b) Superposition of dipole and quadrupole flow. (c) Superposition of dipole, quadrupole and Taylor flow. (d) Superposition of dipole and Taylor flow. Parameters are chosen such that $\lambda=V_{d}=1, V_{t}=0.6, V_{q}=0.4$.

nonuniformity of the temperature gradient and of the proximity of the droplet to the substrate/air interface. For instance, a quadratic nonuniformity in the direction of the primary gradient, $T=\kappa_{0} x+$ $\kappa_{1} x^{2}$, leads to a quadrupole correction (2) to the dipole flow with the same axis $\mathbf{e}=\mathbf{e}_{x}$. In this case the resulting steady flow is still axisymmetric and so again possesses two invariants. The stream function of the combined flow allows us to easily find them:

$$
\begin{aligned}
& \phi=\frac{z}{y}, \\
& \psi=\left(V_{d} a+2 V_{q} x\right) z^{2}\left(a^{2}-r^{2}\right) .
\end{aligned}
$$

The addition of time-periodicity induced by switching the direction of the temperature gradient in the horizontal plane destroys both invariants (15) for almost all values of $\phi$ and $\psi$. As a result, the streamlines are no longer constrained to two-dimensional surfaces and thoroughly sample the droplet volume as the Poincaré section presented in Fig. 7b shows. However, a surface defined by $\phi=\psi=0$, namel, the mid-plane $z=0$, remains invariant with respect to rotations in the horizontal plane and prevents mixing between the top and bottom hemisphere of the 
microdroplet: the streamlines of the flow and hence the trajectories of the dye particles never cross the $z=0$ mid-plane. ( $\psi$ also vanishes at the microdroplet's surface $r=a$, thereby representing a barrier to transport into and out of the droplet.)

This final barrier is destroyed by the correction to the dipole/quadrupole flow induced by the shear in the substrate fluid. Unlike the dipole and quadrupole flows whose streamlines do not cross the mid-plane $z=0$ (see Figs. 1 and 2), the streamlines of the Taylor flow do cross that plane (see Fig. 3). As a result, the streamlines of the combined dipole/quadrupole/Taylor flow can also cross the mid-plane, enabling mixing between the top and bottom hemisphere as the respective Poincaré section illustrates (see Fig. 7c).

It is important to note that the quadrupole flow contribution is essential for introducing chaotic advection in three dimensions. This can be seen by considering the combination of the dipole and Taylor flow alone. Since Taylor flow has the same symmetry with respect to the plane $x=0$ as the dipole flow (compare Figs. 1 and 3) and both flows are timereversible (as is every Stokes flow), all trajectories of the Taylor flow are closed. This, in turn, means that Taylor flow also possesses two invariants. These invariants, however, are not easy to find as Taylor flow is not axially symmetric and hence one cannot use the stream function of the flow. The invariants can nevertheless be found directly from their definition, $\mathbf{v}_{t} \cdot \nabla \psi_{t}=0$. Solving the resulting partial differential equation one finds

$$
\begin{aligned}
& \phi_{t}=y^{3}\left(a^{2}-r^{2}\right), \\
& \psi_{t}=\left(\lambda+1+2 z^{2}-r^{2}\right)^{3}\left(a^{2}-r^{2}\right)^{2} .
\end{aligned}
$$

Again one observes that while the first flow invariant is destroyed by rotations in the horizontal plane, the second invariant is preserved. This is a consequence of a general statement which can be easily proven: any time-reversible flow $\mathbf{v}$ such that

$$
\begin{aligned}
& v_{x}(-x, y, z)=v_{x}(x, y, z), \\
& v_{y}(-x, y, z)=-v_{y}(x, y, z), \\
& v_{z}(-x, y, z)=-v_{z}(x, y, z)
\end{aligned}
$$

possesses at least one invariant which is preserved under rotations in the $x y$ plane. This statement follows directly from the symmetry of the closed orbits of such flows. In particular, the combination of the dipole and Taylor flow has an invariant which is preserved under rotations. In the limit $\lambda \rightarrow \infty$ it takes a simple form:

$$
\psi=\left(V_{t}-V_{d} z\right)^{2}\left(a^{2}-r^{2}\right) .
$$

The corresponding time-periodic flow whose direction is switched in the horizontal plane generates a chaotic invariant set which again lies on a twodimensional surface of revolution defined by the remaining invariant. The cross section of such a surface is shown in Fig. $7 \mathrm{~d}$. This result proves the numerical conclusion of Bryden and Brenner ${ }^{24}$ that the superposition of the dipole and Taylor flow is non-chaotic when the vorticity vector of the shear flow is orthogonal to the axis of the dipole flow.

To summarize, for the case of a thermocapillarydriven microdroplet floating in an immiscible liquid, complete three-dimensional mixing can be obtained by including three ingredients to destroy all invariants: (a) flow time-dependence (e.g., via direction switching in a plane), (b) nonuniformity in the temperature gradient, and (c) circulation due to external shear flow (with vorticity perpendicular to the direction of the temperature gradient). If any of these ingredients is missing, mixing is incomplete. For instance, inclusion of (c) alone leads to non-chaotic flow preserving two invariants while the addition of (a) destroys one of the invariants, leading to chaotic mixing on two-dimensional surfaces. We have seen above that inclusion of (a) and (b) alone is insufficient to produce full three-dimensional mixing. As another illustration, if the time-dependence is due to a periodic modulation of the strength of nonuniformity ${ }^{25}$ one again obtains chaotic mixing in two dimensions as the invariant $\phi$ in (15) is preserved. Finally, inclusion of (b) and (c) alone also preserves one invariant leading to a quasi-periodic flow whose streamlines lie on two-dimensional surfaces. The structure of the flow field in the latter case is qualitatively similar to that of the steady dipole flow shown in Fig. 6a-c: the flow originating near the surface forms a narrow "tube" joining the poles and returns along the surface of the droplet.

The proximity of the microdroplet to the substrate interface can lead to additional corrections to the flow inside the droplet. However, according to our analytical and numerical calculations these corrections do not change the symmetry of the flow and hence the number of invariants, so the essential results of the model described above should still apply.

\section{Experiments}

To test the theory, we conduct experiments using the setup described previously. The mixing experiments begin by merging a nanoliter-scale droplet dyed with $0.5 \mu \mathrm{m}$ diameter fluorescent microspheres with a microliter-scale undyed droplet. Without further manipulation, diffusion would govern mixing. 

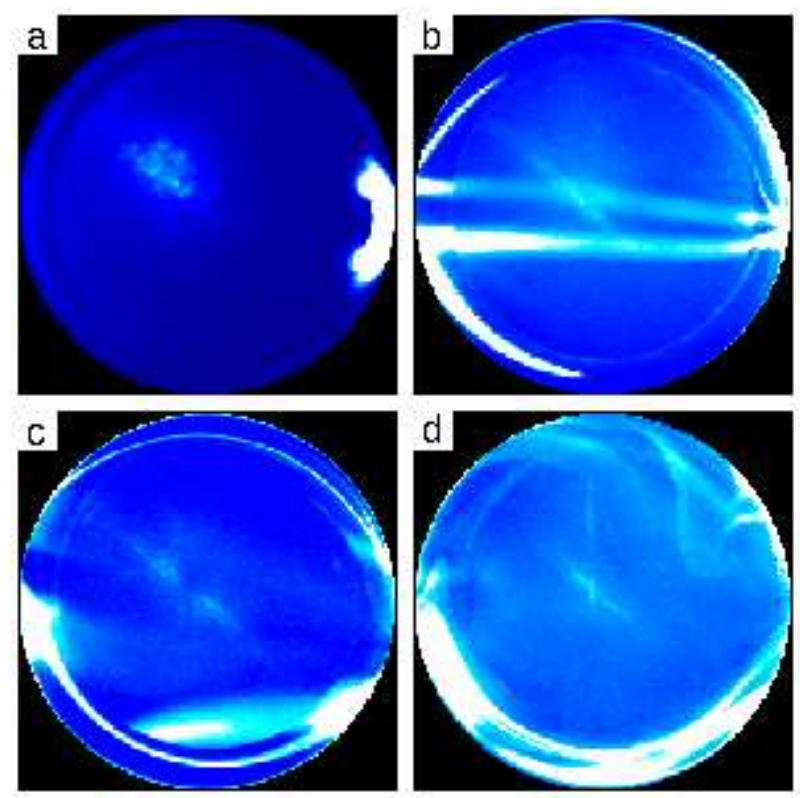

Figure 8: Video images under monochromatic illumination at $488 \mathrm{~nm}$ illustrating optically controlled driving of an aqueous microdroplet in a single direction. (a) A 30 nanoliter droplet dyed with fluorescent microspheres is merged with a 3.7 microliter $(0.96 \mathrm{~mm}$ diameter) undyed droplet. The combined droplet is subsequently driven in one direction and imaged after traveling a distance of (b) $1.0 \mathrm{~cm}$, (c) $2.0 \mathrm{~cm}$ and (d) $3.5 \mathrm{~cm}$.

With a diffusion coefficient for the microspheres of $D=7 \times 10^{-10} \mathrm{~cm}^{2} / \mathrm{s}$ as determined from the StokesEinstein equation, the timescale required for diffusive mixing is $\sim 100$ days. After merger, the combined droplet is driven with an average characteristic speed of $V \sim 0.1 \mathrm{~cm} / \mathrm{s}$. (Higher droplet speeds of a few $\mathrm{cm} / \mathrm{s}$ are possible with this system.). As a result, the droplet flow is characterized by low Reynolds number $\sim 0.1$ and high Péclet number $\sim 10^{7}$, placing the system in a laminar flow regime where mixing is expected to be particularly difficult.

Time series of images of driven droplets demonstrate directional changes in the droplet motion strongly enhance mixing. After merger, the fluorescent microspheres are localized in a small region near the surface of the combined droplet (Figs. 8a and $9 a$ ). If the droplet is subsequently driven in a single direction, the dye is stretched along a "tube" joining the poles and then around the perimeter of the droplet, but does not penetrate the droplet interior (Fig 8b-d). This is consistent with the theoretical analysis of the superposition of dipole, quadrupole, and Taylor flows. In contrast, by periodically changing directions using the same protocol employed in the model, the dye is quickly advected through-
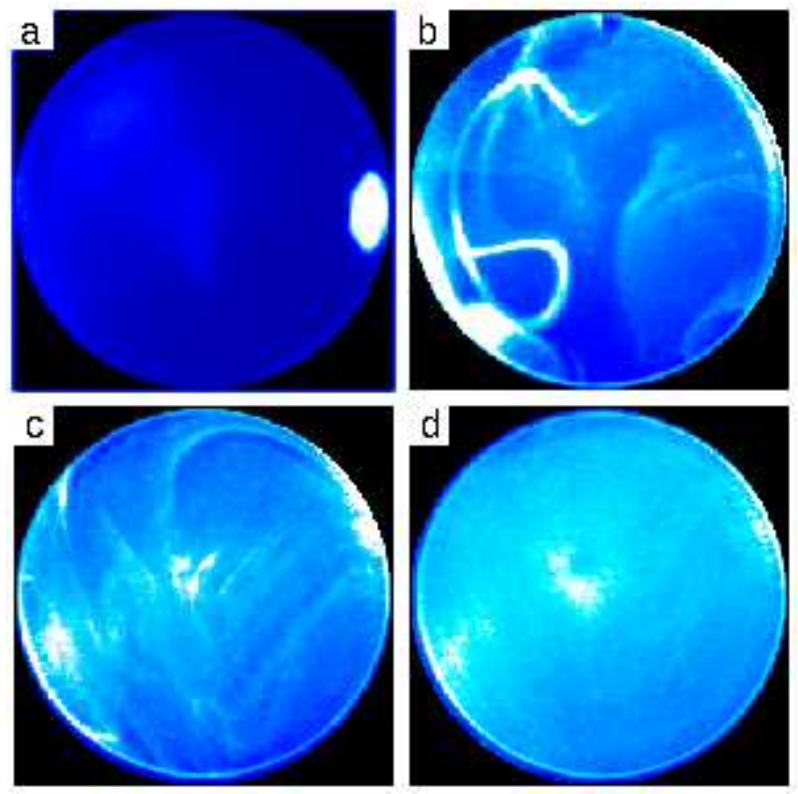

Figure 9: Video images illustrating optically controlled driving of an aqueous microdroplet along a path that periodically changes direction. (a) A 10 nanoliter droplet dyed with fluorescent microspheres is merged with a 4.5 microliter (1.02 mm diameter) undyed droplet. The combined droplet is subsequently driven repeatedly along a square path with side length $3.5 \mathrm{~mm}$ and imaged after changing directions (b) 4 times (c) 12 times and (d) 20 times (for a total path length of $7 \mathrm{~cm}$ ).

out the entire droplet volume (Fig. 9b-d). For a sufficient number of direction changes, the droplet is fully mixed, as verified by illuminating different planes within the droplet with a $100 \mu$ m-thick laser sheet (not shown).

\section{CONCLUSIONS}

The analysis presented above should have broad applicability. On the most general level, one concludes that the existence and number of invariants play a crucial role in determining the mixing properties of the flow. In particular, in order to achieve full three-dimensional mixing the flow within the droplet should be designed to destroy all invariant surfaces in the interior of the droplet. As the example with the dipole/Taylor flow combination shows, destroying the simple geometrical symmetries of the flow might not be sufficient for complete mixing. In this sense the flow invariants provide a more useful way of characterizing the mixing properties of the flow than its symmetries.

More specifically, the types of flows that we have considered (dipole, quadrupole, and Taylor flow) 
are the most common types of interior flows arising inside spherical liquid microdroplets, regardless of the nature of driving forces, so many details of our analysis should be directly applicable to situations when microflows are driven by, e.g., external shear, buoyancy, or electrical fields. Furthermore, many of the results we have obtained for spherical droplets should naturally generalize to deformed shapes such as plugs in microchannels with cylindrical or rectangular cross-section ${ }^{27,32}$ or droplets squashed between two parallel planes. ${ }^{29-31}$ The flows inside these shapes are topologically similar to flows in spherical microdroplets and therefore should have similar mixing properties, at least in steady state.

Finally, our results suggest that optically controlled thermocapillary actuation can be used as a general approach to performing many basic microfluidic operations such as moving, merging and mixing liquid microdroplets. Today's mainstream approach to microfluidics uses lithography-based methods (inspired by microelectronics manufacture) to build networks of microchannels, micropumps, and microvalves. The opto-microfluidic approach employed in this study should permit complex fluidic operations to be performed with no moving parts and no need to construct on-chip devices, sidestepping unsolved problems of the conventional approach (e.g., clogging of microchannels). Opto-microfluidics can also enable, in principle, a single device to be "reprogrammed" to perform different fluidic operations in different possible sequences - yielding, in effect, a microfluidic processing unit $(\mu \mathrm{PU})$. Widespread implementation of this alternative approach to microfluidics has the potential for radically transforming current thinking about how practical devices might be built.

Support for this work by the National Science Foundation and the Research Corporation is gratefully acknowledged.

\section{References}

[1] G. M. Whitesides and A. D. Stroock, "Flexible methods for microfluidics," Phys. Today 54, 4248 (2001).

[2] L. Bousse, C. Cohen, T. Nikiforov, A. Chow, A. R. Kopf-Sill, R. Dubrow and J. W. Parce, "Electrokinetically controlled microfluidic analysis systems," Annu. Rev. Biophys. Biomol. Struct. 29, 155-181 (2000).

[3] M. Burns, C. Mastrangelo, T. Sammarco, F. Man, J. Webster, B. Johnson, B. Foerster, D.
Jones, Y., Fields, A. Kaiser, and D. Burke, "Microfabricated structures for integrated DNA analysis," Proc. Nat. Acad. Sci. USA 93, 55565561 (1996).

[4] T. S. Sammarco and M. A. Burns, "Thermocapillary pumping of discrete drops in microfabricated analysis devices," AIChE J. 45, 350-366 (1999).

[5] H. Aref, "Stirring by chaotic advection," J. Fluid Mech. 143, 1-21 (1984).

[6] J. M. Ottino, "The kinematics of mixing: stretching, chaos, and transport" (Cambridge Univ. Press, Cambridge, 1989).

[7] R. H. Liu, M. A. Stremler,K. V. Sharp, M. G. Olsen, J. G. Santiago, R. J. Adrian, H. Aref, and D. J. Beebe, "Passive mixing in a threedimensional serpentine microchannel," J. Microelectromech. 9, 190-197 (2000).

[8] A. D. Stroock, S. K. W. Dertinger, A. Ajdari, I. Mezic, H. A. Stone, and G. M. Whitesides, "Chaotic mixer for microchannels," Science 295, 647-651 (2002).

[9] A. A. Darhuber, J. P. Valentino, J. M. Davis, S. M. Troian, and S. Wagner, "Microfluidic actuation by modulation of surface stresses," Appl. Phys. Lett. 82, 657-659 (2003).

[10] M. G. Pollack, R. B. Fair and A. D. Shenderov, "Electrowetting-based actuation of liquid droplets for microfluidic applications," Appl. Phys. Lett. 77, 1725-1726 (2000).

[11] S. K. Cho, H. Moon and C.-J. Kim, "Creating, transporting, cutting, and merging liquid droplets by electrowetting-based actuation for digital microfluidic circuits," J. Microelectromech. 12, 70-80, (2003).

[12] J. S. Hadamard, "Mouvement permenent lent d'une sphere liquide et visqueuse dans un liquide visqueux," C. R. Acad. Sci. Paris 152, 1735-1538 (1911).

[13] N. O. Young, J. S. Goldstein and M. J. Block, "The motions of bubbles in a vertical temperature gradient," J. Fluid Mech. 6, 350-356 (1959).

[14] S. M. Lee, D. J. Im and I. S. Kang, "Circulating flows inside a drop under time-periodic non-uniform electric fields," Phys. Fluids 12, 1899-1910 (2000). 
[15] G. I. Taylor, "The viscosity of a fluid containing small drops of another fluid," Proc. R. Soc. London A 138, 41-48 (1932).

[16] G. I. Taylor, "Studies in electrohydrodynamics I. The circulation in a drop by an electric field," Proc. R. Soc. London, Ser. A 291, 159166 (1966).

[17] I. Mezic and S. Wiggins, "On the integrability and perturbation of three-dimensional fluid flows with symmetry," Nonlinear Sci. 4, 157-194 (1994).

[18] D. L. Vainshtein, A. A. Vasiliev and A. I. Neishtadt, "Changes in the adiabatic invariants and streamline chaos in confined incompressible Stokes flow," Chaos 6, 67-77 (1996).

[19] A. I. Neishtadt, D. L. Vainshtein and A. A. Vasiliev, "Chaotic advection in a cubic Stokes flow," Physica D 111, 227-242 (1998).

[20] D. Kroujiline and H. A. Stone, "Chaotic streamlines in steady bounded threedimensional Stokes flows," Physica D 130, 105-132 (1999).

[21] J. R. Angilella and J. P. Brancher, "Note on chaotic advection in an oscillating drop. Phys. Fluids," 15, 261-264 (2003).

[22] K. Bajer and H. K. Moffatt, "On the class of steady confined Stokes flows with chaotic streamlines," J. Fluid Mech. 212, 337-363 (1990).

[23] H. A. Stone, A. Nadim, and S. H. Strogatz, "Chaotic streamlines inside drops immersed in steady Stokes flows," J. Fluid Mech. 232, 629646 (1991).

[24] M. D. Bryden and H. Brenner, "Mass-transfer enhancement via chaotic laminar flow within a droplet," J. Fluid Mech. 379, 319-331 (1999).

[25] T. Ward and G. M. Homsy, "Electrohydrodynamically driven chaotic mixing in a translating drop," Phys. Fluids 13, 3521-3525 (2001).

[26] T. Ward and G. M. Homsy, "Electohydrodynamically driven chaotic mixing in a translating drop. II. Experiments," Phys. Fluids 15, 29872994 (2003).

[27] K. Hosokawa, T. Fujii and I. Endo, "Handling of picoliter liquid samples in a poly(dimethylsiloxane)-based microfluidic device," Anal. Chem. 71, 4781-4785 (1999).
[28] P. Paik, V. K. Pamula, M. G. Pollack and R. B. Fair, "Electrowetting-based droplet mixers for microfluidic systems," Lab Chip 3, 28-33 (2003).

[29] P. Paik, V. K. Pamula, M. G. Pollack and R. B. Fair, "Rapid droplet mixers for digital microfluidic systems," Lab Chip, advance article (2003).

[30] J. Fowler, H. Moon and C.-J Kim, "Enhancement of mixing by droplet-based microfluidics," Proc. IEEE Conf. MEMS, Las Vegas, 2002, p. 97-100.

[31] T. Taniguchi, T. Torii and T. Higuchi, "Micro chemical reactor in micro droplets - electrostatic manipulation of micro droplets," Proc. ISMM 2001, p. 104-105.

[32] H. Song, J. D. Tice and R. F. Ismagilov, "A microfluidic system for controlling reaction networks in time," Angew. Chem. Int. Ed. 42, 768772 (2003).

[33] D. E. Kataoka and S. M. Troian, "Patterning liquid flow on the microscopic scale," Nature 402, 794-797 (1999).

[34] N. Garnier, R. O. Grigoriev and M. F. Schatz, "Optical manipulation of microscale fluid flow," Phys. Rev. Lett. 91, 054501 (2003).

[35] E. Fuchs, J. S. Jaffe, R. A. Long and F. Azam, "Thin laser light sheet microscope for microbial oceanography," Optics Express 10, 145-154 (2002).

[36] H. Brenner, "The Stokes resistance of a slightly deformed deformed sphere," Chem. Engng. Sci. 19, 519-539 (1964).

[37] S. H. Chen, "Movement of a fluid sphere in the vicinity of a flat plane with constant temperature gradient," J. Colloid Interf. Sci. 230, 157170 (2000).

[38] M. Meyyappan and R. S. Subramanian, "Therocapillary migration of a gas bubble in an arbitrary direction with respect to a plane interface," J. Colloid Interf. Sci. 115, 206-219 (1987). 